\title{
PRIMERA ETAPA DEL PROYECTO DE INVESTIGACIÓN “LAS RUTINAS DIARIAS Y EL BALANCE OCUPACIONAL DE LOS ALUMNOS DE LA UNIVERSIDAD NACIONAL DE QUILMES"
}

\author{
FIRST STAGE OF THE RESEARCH PROJECT "DAILY ROUTINES AND OCCUPATIONAL \\ BALANCE OF STUDENTS FROM THE NATIONAL UNIVERSITY OF QUILMES"
}

\section{Demiryi, Milagros'; Berezin, Silvia²; Fernández, Graciela³; Ganso, Héctor; Gaiteiro, Adriana $^{5}$}

\section{Resumen:}

Existen argumentos teóricos y antecedentes de investigación que prueban la relación entre las rutinas diarias y las tensiones que afectan la salud y el bienestar.

En el diario vivir las personas respondemos de manera cada vez más acelerada a necesidades y exigencias ocupacionales laborales, de estudio y del hogar. El estrés y el síndrome de la mente en carrera son ejemplos típicos de algún desajuste.

Las Universidades se han estudiado a sí mismas desde aspectos históricos, políticos, didácticos y problemáticas de los estudiantes como la deserción temprana y tardía y el desempeño académico. Las rutinas diarias y las percepciones que los alumnos tienen del balance ocupacional es un tema de estudio poco desarrollado.

La presente investigación acreditada por la Universidad Nacional de Quilmes se encuentra en la etapa inicial. Se realizará una investigación cualitativa con enfoque fenomenológico, de tipo Investigación-Acción Participativa que propiciará la participación de los alumnos de la Universidad

Se espera lograr la producción de conocimientos que permitan caracterizar las rutinas diarias y la percepción del balance ocupacional de los alumnos para la detección temprana de indicadores de desbalance ocupacional y la elaboración de propuestas para su afrontamiento, lo que reducirá eventuales costos de salud.

Palabras clave:

Rutinas diarias, equilibrio ocupacional, estudiantes.

1 Terapista Ocupacional. Especialista en Docencia Universitaria, Especialista en Docencia en Entornos Virtuales. Prof. Adjunto. Docenteinvestigadora UNQ-UNL. San Juan 2438, CP3000, Santa Fe, Argentina. TE: 0342-155035916. mdemiryi2@uvq.edu.ar

2 Licenciada en Terapia Ocupacional. Magíster en Gerontología. Prof. Adjunto. Docente-investigadora UNQ. Alvear 90, CP 6740, Chacabuco, Bs., As, Argentina. TE: 02352 427668. sberezin@unq.edu.ar

3 Licenciada en Matemáticas. Magíster en Ciencias Sociales. Profesora Adjunta y Asociada, Docente-investigadora de UNQ y UNLaM. Moreto № 518 / 520, C. P. 1407, Bs.As. Te: 011- 46713182.gfernandez14@uvq.edu.ar grafernandez7@yahoo.com.ar

4 Médico-Pediatría, Neonatología y Neurología infantil. Profesor Titular. Docente-investigador UNQ. Avenida Mitre 2300,Piso 8, Dto A, Avellaneda, CP 1872, Bs As. TE: 011- 1531413091. hectorganso@ciudad.com.ar

5 Lic. en Terapia Ocupacional, Becaria de Investigación UNQ. Bragado 6039, Dto.3, Wilde, Avellaneda, CP 1875, Bs.As. TE: 011-42067703. adrianagaiteiro@hotmail.com 


\begin{abstract}
Abstrac:
There are theoretical arguments and research background to prove the relationship between daily routines and tensions that affect health and well-being.

In the daily life people respond increasingly accelerated to needs and work, occupation, study and home demands. Stress and the career mind syndrome are typical examples of some imbalance.

Universities have studied themselves from historical, political and didactic aspect and students problematic such as: early and late desertion and academic performance.

Daily routines and the students perception of the occupational balance, it is a slightly developed topic.

This research, accredited by the National University of Quilmes, is now in the initial stage. It is going to be a qualitative research, with phenomenological approach, a participatory action research - type, to encourage the students from the University to participate.

It hopes to achieve the production of knowledge to characterize the daily routines and the students occupational balance perception, for the early detection of indicators of occupational imbalance and the elaboration of proposals to confront it, which will reduce any health costs.
\end{abstract}

\title{
Keywords:
}

Daily routines, occupational balance, students.

\section{INTRODUCCIÓN:}

Existen argumentos teóricos y suficientes antecedentes de investigación que prueban la relación entre las rutinas diarias y las tensiones que afectan la salud y el bienestar.

El diario vivir transcurre en un tiempo que se presenta cada vez más acelerado y en el cual las personas debemos satisfacer nuestras necesidades y responder a exigencias relacionadas con ocupaciones laborales, de estudio, del hogar, entre otras. El estrés y el síndrome de la mente en carrera son ejemplos típicos de algún desequilibrio.

Se asume que equilibrar la vida es bueno para la salud. Los estudios sobre equilibrio ocupacional necesitan de mayor sustento teórico y evidencias empíricas producidos en diferentes contextos ocupacionales socio-culturales.

Las experiencias desarrolladas permiten afirmar que la ocupación y en particular aquellas que resultan significativas, poseen potencial sinérgico y aportan a los estados de salud y bienestar. Que las personas definen su sentirse saludables asociando las cosas que pueden hacer y que resultan placenteras. Que los contextos aparecen en muchas ocasiones como limitantes y perjudiciales.

La vivencia de presiones en la vida diaria se asocia a desequilibrio, cuando las exigencias exceden los recursos personales y contextuales. Muchas veces estas situaciones obligan a las personas a realizar ajustes y adaptaciones ocupacionales que pueden tener carácter temporal o circunstancial (determinadas por los cam- bios que fuerzan a realizar las ocupaciones de otra forma, a hacer algunas ocupaciones y otras no) o ser adaptaciones estables que permanecen en el tiempo.

La superposición de ocupaciones, la recarga ocupacional o sobreocupación y la orquestación-coordinación de ocupaciones son fenómenos que se producen en muchos casos, cuando las personas pierden el control y capacidad de tomar decisiones ocupacionales. Las dinámicas de trabajo y de algunas instituciones, así como otras características de los entornos, contribuyen a sobrecargar innecesariamente las rutinas diarias.

La falta de preparación para determinadas situaciones de cambio en la vida de las personas, como pueden ser: la jubilación, las enfermedades y catástrofes, producen desequilibrios en las rutinas.

Las Universidades se han estudiado a sí mismas desde aspectos históricos, políticos, didácticos y problemáticas específicas de los estudiantes, en particular la deserción temprana y tardía. No se han encontrado antecedentes de investigación sobre las características de las rutinas diarias y las percepciones que los alumnos tienen del balance ocupacional.

\section{Antecedentes:}

La sociedad moderna se caracteriza por la cantidad, variedad y vertiginosidad de los cambios que impactan y se expresan de un modo particular en la vida cotidiana. La investigación acumulada en años afirma la tendencia en aumento de la vida estresante (Backman, 2000, Ro- 
binson y Godby, 1997) y las consecuencias perjudiciales para la salud y el bienestar de las personas. En correlato con estas evidencias, desde diferentes disciplinas y perspectivas se han elaborado e implementado propuestas de afrontamiento que en términos generales coinciden en la necesidad de producir un equilibrio de vida. Aun desde este consenso, el alcance y la comprensión de lo que constituye el equilibrio adquiere múltiples significados y es tema de estudio.

En la terapia ocupacional, desde los inicios de la profesión se empleó el término para denominar "los ritmos cotidianos de trabajo, juego, sueño y descanso" (Meyer, A. 1992), considerado la base para una vida saludable.

Los beneficios de la participación en ciertas ocupaciones, para la salud y la calidad de vida (Christiansen y Matuska, 2004), así como las relaciones entre el estrés, los estilos de vida y el bienestar, son ampliamente aceptados, aun reconociendo la necesidad de mayor evidencia empírica. Las ocupaciones son claves de y para la condición y naturaleza humana. Proporcionan organización, estructura, sentido y propósito al quehacer diario y a lo largo de la vida. Los patrones ocupacionales son uno de los dominios de conocimiento de la profesión relacionados a las actividades de la vida diaria que son significativas y habituales para las personas y sus entornos. Un componente e indicador de estos patrones lo constituyen las rutinas, en tanto secuencias establecidas de ocupaciones que proveen una estructura a la vida diaria (Clark, 2000), (Neistadt y Crepeau, 1998), requieren tiempo y se integran en los contextos cultural y ecológico (Fiese et al., 2002). Pueden ser gratificantes o perjudiciales. El uso del tiempo en las ocupaciones diarias valoradas que proveen satisfacción, configuran patrones ocupacionales relacionados con el equilibrio ocupacional. (Eklund, Mona y Erlandsson, LenaKarin y Leufstadius, Christel, 2010).

Algunos autores sostienen que la condición previa al logro del equilibrio es la adaptación y otros lo han utilizado de modo intercambiable. No menos controvertido por su alcance y polisemia, su mención está relacionada a los aportes del estudio de Gómez Lillo, S. (2006) que rastrea los antecedentes previos al surgimiento de la ciencias de la ocupación, en particular las investigaciones sobre distribución del tiempo y equilibrio ocupacional.

El concepto continúa siendo foco de preocupación en la actualidad. Wilcock y Towsend (2008), plantean que el desequilibrio ocupacional puede ocurrir tanto por sobre-ocupación como por sub-ocupación, como consecuencia de situaciones de apartheid y/o injusticia ocupacional. Considerando que es un factor de riesgo para la salud del individuo y de la comunidad. Las estructuras ocupacionales de la sociedad (escuelas, lugares de trabajo, de recreación) y sus políticas generan ambientes ocupacionales que inciden en la salud y el bienestar. Pentland, W. y Mc Coll (2009)' proponen el modelo de "integridad ocupacional" para denominar cuando la mayoría de las ocupaciones son congruentes con los valores, los significados y se encuentran en armonía con el entorno. El aporte de Jonsson, $\mathrm{H}$. $(2006,2007)^{11}$ sobre la experiencia de equilibrio ocupacional consiste en un modelo tridimensional con dinámica interdependiente en sus relaciones y con atributos positivos y negativos. Hakansson, C. $(2006,2011)$ por su parte, ha centrado sus estudios en los aspectos estructurales y experienciales que promueven el balance de vida. Matuska, K. y Christiansen, C. (2004, 2010) han elaborado una propuesta de modelo teórico denominada "balance de vida", basada en la investigación interdisciplinaria sobre los atributos fisiológicos y psicológicos considerados importantes para el bienestar. El balance se conceptualiza en función de cómo las configuraciones de las actividades cotidianas satisfacen las necesidades humanas esenciales.

Sumarizando, el equilibrio ocupacional sigue siendo un concepto polémico y en construcción. Los interrogantes que genera, tal como los enigmas e implicancias, concita interés y convoca voluntades, tal como quienes afirman que: "el estudio del equilibrio de vida puede ser oportuno y útil para la comprensión de los componentes necesarios para reducir el estrés crónico y mejorar la salud y el bienestar" (Matuska, K. 2009).

Existe una percepción compartida de que los tiempos se han acelerado. Las demandas de las ocupaciones laborales, de estudio, del hogar, por mencionar sólo algunas, generan tensiones con efecto acumulativo en la salud. El estrés es una realidad de la sociedad en que vivimos. Nos encontramos ante una problemática que merece atención.

Como miembros de la comunidad académica de la Universidad Nacional de Quilmes, vivimos estas tensiones y las evidenciamos a diario en nuestros alumnos. La vida en las grandes ciudades como Buenos Aires, acentúa las características antes mencionadas. Cómo organizan y vivencian sus rutinas ocupacionales y el sentido de equilibrio la población estudiantil de la Universidad, son los interrogantes que motivan la presente investigación.

La búsqueda de respuestas a un problema teórico de actualidad, con anclaje en un segmento poblacional en formación como futuro recurso profesional, es una 
apuesta y un desafío a combinar acciones de docencia e investigación; escenario en el que se pretende potenciar las funciones de la Universidad en la construcción activa y transferencia de conocimientos desde y para mejorar la salud de los propios actores.

\section{Objetivos que se pretenden alcanzar}

- Realizar un aporte a la salud y bienestar ocupacional de los estudiantes de la UNQ.

- Caracterizar las rutinas diarias de los estudiantes de la UNQ.

- Detectar la percepción que tienen los estudiantes sobre el balance de sus ocupaciones.

- Identificar los factores que amenazan el sentido de satisfacción y bienestar.

- Elaborar propuestas que contribuyan a su afrontamiento.

\section{Metodología}

Se realizará una investigación cualitativa con enfoque fenomenológico, de tipo Investigación-Acción Participativa.

Desde la perspectiva epistemológica se pondera el conocimiento experiencial y la subjetividad en la investigación.

Desde la perspectiva ontológica se requiere la participación de los actores involucrados para la construcción de conocimientos a través de la reflexión de las propias experiencias.

Esta metodología potencia el carácter educativo de la investigación como medio de empoderamiento. Implica un proceso de compartir información colaborativa, investigación sistemática, ciclos de reflexión, acción y modificación. Son de utilidad para los profesionales interesados en hacer que su práctica sea colaborativa y centrada en las necesidades de las personas además del desafío de proporcionar servicios basados en evidencias.

Se realizará selección no probabilística de los participantes. Propiciando la participación de los alumnos de todas las carreras de la UNQ, mediante información previa del presente proyecto y sus objetivos, por medio de las autoridades pertinentes.

El trabajo se iniciará con los alumnos de la Licenciatura en Terapia Ocupacional en las asignaturas cuyos contenidos se relacionan con el tema de estudio. En particular: Teorías de Terapia Ocupacional, Prácticas Profesionales e Investigación en Terapia Ocupacional.

Con los alumnos de las demás carreras que manifiesten su consentimiento para participar, se organizarán talleres teórico-prácticos sobre el tema. Se prevé como mínimo dos encuentros por grupo.

Se revisarán los instrumentos de evaluación de rutinas diarias y equilibrio ocupacional disponibles.

Se seleccionarán aquellos que mejor resulten para recabar la información que se pretende producir. $\mathrm{Si}$ fuera necesario, se adaptarán y/o construirán nuevos instrumentos. Se prevé utilizar para la recolección de datos: observaciones participantes, entrevistas cualitativas, talleres y situaciones áulicas. Se incluirán procedimientos tales como filmación, grabación y fotografías.

El análisis de los datos se producirá en paralelo a la recolección de modo tal que lo producido en el primer encuentro grupal, sea devuelto como insumo para la reflexión en el siguiente encuentro de manera sucesiva. Se aplicará triangulación de técnicas y de investigadores. Estas estrategias en combinación con los investigadores participantes, el examen de pares y el registro mecánico de los datos se utilizarán para aumentar la validez interna del estudio.

\section{Expectativas}

Se espera lograr la producción de conocimientos que permitan identificar las características de las rutinas diarias y la percepción del balance ocupacional de los alumnos de la UNQ.

El tipo de diseño propuesto permitirá captar una información que no está disponible, sobre una temática de impacto en la vida ocupacional y el desempeño de los estudiantes. Constituirá un aporte tanto para la evidencia empírica como para los constructores teóricos y las líneas de acción.

Los resultados se constituirán en insumos básicos para introducir los cambios necesarios en el sistema educativo, en sus políticas, en la organización y modalidades de la oferta académica.

Permitirá ponderar el rol de la Universidad en la educación y la investigación de problemáticas ocupacionales de actualidad que potencialmente afectan la salud y el bienestar de los futuros profesionales en formación, contribuyendo a elevar la calidad de los recursos humanos. 
La detección temprana de indicadores de disbalance ocupacional y la elaboración de propuestas para su afrontamiento reducirá eventuales costos en salud.

\section{Impacto del Proyecto}

El diseño de investigación propuesto se articula con las acciones de docencia y conlleva la participación activa de los estudiantes de la comunidad educativa en formación.

Este proyecto implica centralmente que los alumnos al ser sujetos de conocimiento, se iniciarán tempranamente en acciones de investigación para la resolución de problemas cotidianos. La investigación se presenta como una ocupación viable, de aplicación directa y uso para la práctica profesional.

Se recorrerá un espectro que va desde la formación de grado para alumnos de la carrera de terapia ocupacional hasta la iniciación en la investigación para docentes, graduados y alumnos avanzados.

La universidad, al asumir la responsabilidad de temas de salud y calidad de vida de los estudiantes, se constituye en promotor de la misma. De este modo, los conocimientos producidos en la academia podrán ser tomados como base para su instalación en la agenda pública y para la toma de decisiones políticas.

\section{RESULTADOS PRELIMINARES}

La revisión de instrumentos de evaluación de rutinas y equilibrio ocupacional arrojó como resultado la existencia de instrumentos producidos y en proceso de validación en el exterior (Matuska \& Christiansen, 2008), la limitada producción en nuestro país y Latinoamérica y la percepción de la utilización de instrumentos desactualizados. Estos últimos elementos concurren en la detección de un tema de vacancia que abrió una línea de investigación complementaria. Iniciativa que se desarrolla en el marco del presente proyecto por medio de una Beca de Formación en Docencia e Investigación del Departamento de Ciencias Sociales de la Universidad Nacional de Quilmes.

Se confeccionó un instrumento de evaluación de rutinas diarias y percepción de balance ocupacional, organizado en las siguientes categorías: - Actividades según áreas ocupacionales, - Tiempo en horas, - Dificultades, - Satisfacción según escala, - Indicadores psi- cofísicos de estrés, - Propuestas e -Identificaciones conceptuales. El formato es el de un Cuestionario con 25 ítems que combina preguntas cerradas y dicotómicas con preguntas abiertas. Los aportes del Modelo Teórico de Conciliación de la Vida (LBM) y el Marco de Trabajo para la Práctica de Terapia Ocupacional se utilizaron para la validez de contenido.

Se realizó una prueba piloto con una muestra de conveniencia de 87 alumnos de diferentes cursos de la Lic. en Terapia Ocupacional, en dos momentos, en una primera instancia se procedió a la recogida de datos y en la segunda a la devolución, discusión y puesta en común de los resultados parciales obtenidos. Previo a la implementación del Cuestionario se informó a la Dirección de la Carrera y se difundió el proyecto a todos los docentes, invitándolos a colaborar. De igual modo se procedió con los alumnos participantes que recibieron y firmaron el consentimiento informado elaborado por el equipo. Se les presentó el proyecto y se los instruyó para completar el cuestionario. Durante la recolección se atendieron las consultas que fueron registradas por escrito. Se asumió el compromiso de realizar la devolución de los resultados. El procesamiento estadístico de los datos y la categorización arrojaron los siguientes resultados centrales.

Las actividades de rutina con mayores dificultades según áreas ocupacionales son (presentados en orden de prioridad): -Movilidad (Demasiado tiempo de viaje y de espera del medio de transporte, malas condiciones de los servicios) - Estudio (Muchas horas, especialmente por las prácticas profesionales, problemas con la oferta académica, falta de flexibilidad, presión del tiempo), y -Trabajo (Demasiadas horas, falta de valoración, colisión con las horas de estudio). El factor común es el Tiempo, por exceso o por carencia. El $62 \%$ de los alumnos no se encuentran satisfechos con sus rutinas diarias. El $52 \%$ y más de los alumnos presentan indicadores de trastornos psicofísicos desde el comienzo de la carrera. El 75\% identifica conceptualmente el término equilibrio como más adecuado. Las propuestas presentadas son en relación a: - la movilidad, - lo académico, - actividades a incorporar en la vida universitaria.

En base a las observaciones y respuestas de los encuestados respecto al instrumento, se detectaron limitaciones que motivaron su revisión. Se detectó también la falta de manejo por parte de los alumnos, de elementos propios de la disciplina como el Marco de Trabajo, lo que agregó dificultad para la medición. Se creó la versión modificada que presenta de manera más sintética las categorías de ocupaciones y elimina el elemento 
Dificultades, por no haber resultado válido para la medición. Otras preguntas fueron reformuladas para una mayor claridad.

Para la devolución de los resultados, se realizaron tres talleres, de 2 horas de duración cada uno, en los que participaron 75 alumnos que habían respondido al Cuestionario de Rutinas. Se presentaron los Resultados, Conclusiones preliminares y Propuestas, junto con una Guía temática impresa en papel, construida en base a la información recabada, para orientar la discusión de los hallazgos. La dinámica incluyó el trabajo en pequeños grupos y en plenario. La activa y entusiasta participación de los alumnos en esta instancia, los niveles de reflexión sobre sus propias prácticas así como las propuestas elaboradas, confirman la validez de la metodología escogida en el presente proyecto.

La utilización de las técnicas de grupo focal y observación participante junto con los registros fílmicos, fotográficos y de grabación, permiten disponer de abundante información. Se comenzó el proceso de análisis de los datos obtenidos en los talleres.

El Cuestionario de Rutinas (versión final), se aplicó a otro grupo de 65 alumnos de terapia ocupacional. Los datos recolectados se han comenzado a analizar.

Estos resultados corresponden al primer año de desarrollo del proyecto.

\section{ReferenCias Bibliográficas}

Hakanson, C.; Wagman, P.; Björklund, A. Equilibrio ocupacional: un análisis del concepto que se utiliza en la terapia ocupacional. 2011. Facultad de Ciencias de la Salud, Universidad de Jönköping, Suecia. Scandinavian nJournal of Occupational Therapy. disponible en: http://informahealthcare.com/occ.

Backman, C.; Robinson y Godby. Occupational balance: Exploring the relationships among daily occupations. 2004. The Canadian Journal of Occupational Therapy, 71, 4; CBCA.

Christiansen, C. H. \&Matuska, K. M. The importance of everyday activities. En C. H. Christiansen \& K. M. Matuska (Eds.), Ways of living: Adaptive strategies for special needs. 2004. (3rd ed., pp. 1-20). Bethesda, MD: AOTA Press.

Clark, F. A. The concept of habit and routine: A preliminary theoretical synthesis. Occupational Therapy Journal of Research. 2000. 20, 123S-137S.

Eklund, Mona y Erlandsson, Lena Karin y Leufstadius, Christel. Uso del Tiempo en Relación a las Ocupaciones valoradas y satisfacción entre las personalidades con enfermedad mental per- sistente: Explorando el Equilibrio Ocupacional. 2010. En Journal of Occupational Science, V. 17. Pag. 231-238.

Fiese, B. H.; Tomcho, T. J.; Douglas, M.; Josephs, K.; Poltrock, S. \& Baker, T. A review of 50 years of research on naturally occurring family routines and rituals: Cause for celebration? Journal of Family Psychology. 2002, 16, 381-390.

Gómez Lillo, S. Equilibrio y organización de la rutina diaria. 2006. Revista Chilena de Terapia Ocupacional. Universidad de Chile. Facultad de Medicina. Escuela de Terapia Ocupacional. Nº6. Disponible en: http://www.revistas.uchile.cl/index.php/RTO/ article/viewArticle/111

Hakansson, C.; Dahlin-Ivanoff, S. \& Sonn, U. Achieving balance in everyday life. 2006. Journal of Occupational Science, 13, 74-82.

Jonsson, $\mathrm{H}$. Towards a new direction in the conceptualization and categorization of occupation. 2007. [Wilma West Lecture, Occupational Science Symposium]. Los Angeles: University of Southern California, Occupational Science and Occupational Therapy.

Jonsson, H.; Persson, D. Towards an Experiential Model of Occupational Balance: An Alternative Perspective on Flow Theory Analysis. 2006. Journal of Occupational Science, 13 (1), 62-73.

Matuska, K:, Christiansen, C:, Polatajko, H. \& Davis, J. (Eds.). Conciliación de la vida: Teorías e investigaciones multidisciplinarias. 2009. AOTA Press, Bethesda, MD EE.UU, ISBN 978-1-55642906-4, Capítulo 4, pp. 33-42.

Matuska, K. Validity Evidence for a Model and Measure of Life Balance. 2010. Disponible en: http://conservancy.umn.edu/bitstream/93965/1/Matuska umn 0130E 11207.pdf

Matuska, K.; Heinz, A.; Jonsson, H.; Pentland, W.; Hakansson, C.; Davis, J.; Backman, C. Life balance: Multidisciplinary theories and research. 2010. Presentado en el XV Congreso Internacional de la Federación Internacional de Terapia Ocupacional. WFOT. Stgo. Chile.

Meyer, A. The philosophy of occupational therapy. Archives of Occupational Therapy, 1992 1, 1-10.

Neistadt, M., Crepeau. Tratamiento del estrés. En Hopkins H. y Smith, H. Willard and Spackman. 1998. Terapia Ocupacional. $8^{a}$ Ed. Editorial Médica Panamericana. Unidad VII, 4, 588- 596.

Pentland, W. y McColl. Another perspective on life balance - Living in integrity with values. 2009. En Matuska, K. y Christiansen, C. (Eds.), Life balance: Multidisciplinary research and theories. Bethesda: AOTA Press and Slack, Inc.

Wilcock, A. \& Townsend, E. A. Justicia Ocupacional. 2008. En E. B. Crepeau, E. S. Cohn, \& B. B. Schell (Eds.), Willard and Spackman. Terapia Ocupacional. 11th ed. Editorial Médica Panamericana. Unidad III, 20, 192-199. 